\title{
Compact Urea Sensor System Based on Chemiluminescence for Dialysis Machine
}

\author{
Kunihito Hayashi, ${ }^{1,2}$ Jun'ya Hori, ${ }^{1}$ Masahiro Ozaki, ${ }^{1 *}$ \\ Masuo Nakagawa, ${ }^{1}$ and Masafumi Hashiguchi ${ }^{3}$ \\ ${ }^{1}$ Department of Applied Physics, Graduate School of Science, Okayama University of Science, \\ 1-1 Ridai-cho, Kita-ku, Okayama 700-0005, Japan \\ ${ }^{2}$ Department of Clinical Engineering, Sumida Clinic, \\ 3-3-113 Chuoh, Souja, Okayama 719-1131, Japan \\ ${ }^{3}$ Richo Company, Ltd., 1005 Shimo-Ogino, Atsugi, Kanagawa 243-0298, Japan
}

(Received June 12, 2018; accepted November 9, 2018)

Keywords: spent dialysate, urea sensor, chemiluminescence

As the amount of urea removed from the blood $\left(M_{B}\right)$ is equal to that transferred into the spent dialysate during dialysis treatment, $M_{B}$ can be estimated from the urea concentration in the spent dialysate and the flow rate of the dialysate. There has been a need to develop a sensor for measuring the urea concentration during dialysis treatment instead of using reagents to detect urea in the blood. We have created a prototype of a precise urea sensor for measuring urea concentration in the spent dialysate using chemiluminescence (CL). We have reported an accuracy of $3 \%$ for a CL-based urea sensor system with a syringe-pump-type CL reactor and a photosensor module (PM) as the CL detector. However, problems including the poor mechanical durability of the large reactor unit and the high cost of the CL detector with a PM must still be solved. A compact and low-cost urea sensor that can be installed in a dialysis machine is needed. We have attempted to increase the CL emission so as to measure CL using a low-cost photodiode (PD) and to make a reactor that is highly durable. The urea sensor based on CL due to the reaction of urea with hypobromite has a high precision of $1.9 \%$. It also exhibits good correlation with the chemically measured urea concentrations in Okayama Medical Laboratories, Inc. The CL emitted from the new vortex-flow-type reactor was detected using a low-cost Si PD (S1787-08 Hamamatsu, Japan) and a high-gain current amplifier (7 mV/ $\mathrm{pA}$ ), so that we can measure the urea nitrogen concentrations between 2 and $30 \mathrm{mg} / \mathrm{dL}$. This sensor will be used to realize automatic dialysis machines in the near future.

\section{Introduction}

Gotch and Sargent proposed that the urea concentration in the blood decreases exponentially during dialysis, and the level of dialysis can be prescribed by $K t / V$ (product of urea clearance $K$ and treatment time $t$ divided by volume of body water $V$ ). ${ }^{(1)}$ The urea reduction ratio (URR; the difference between pre- and postdialysis urea levels divided by the predialysis urea level) has

*Corresponding author: e-mail: ozaki@dap.ous.ac.jp

https://doi.org/10.18494/SAM.2019.2026 
also been used to quantify dialysis treatment adequacy. ${ }^{(2)}$ As the amount of urea removed from the blood $\left(M_{B}\right)$ is equal to the amount of urea transferred into the spent dialysate during dialysis treatment, $M_{B}$ can be estimated from the urea concentration in the spent dialysate and the flow rate of the dialysate. Therefore, a sensor for measuring urea concentration in the spent dialysate during dialysis treatment instead of using reagents for detecting urea in the blood serum needs to be developed.

Many studies of a urea sensor for measuring urea in the solutions and blood serum have been proposed. ${ }^{(3-6)}$ However, almost all of these sensors were based on the enzyme reaction of urea, and the stability of the enzyme immobilized on the sensor in the spent dialysate is questionable. Currently, a urea sensor based on UV absorption is commercially available; ${ }^{(7)}$ however, it can only measure the approximate urea concentration because the absorption spectrum of urea overlaps with that of the dialysate. ${ }^{(8)}$ On the other hand, Hu et al. proposed the measurement of urea using the chemiluminescence (CL) of urea and hypobromous acid, and the possibility of nitrogen chemiluminescence as a reactive substance was described. ${ }^{(9,10)}$

We have created a prototype of a precise urea sensor for measuring the urea concentration in the spent dialysate using CL. ${ }^{(11,12)}$ We have reported an accuracy of $3 \%$ for a CL-based urea sensor with a syringe-pump-type CL reactor and a photosensor module (PM) as the CL detector. We also confirmed that the sensor is accurate and stable enough to evaluate the dialysis efficiency quantitatively in the maximum range of variation of urea concentration (17-5 $\mathrm{mg} / \mathrm{dL}$ ) during in vivo dialysis treatment. ${ }^{(12)}$ However, problems regarding the poor mechanical durability of the large reactor unit and the high cost of the CL detector with a photomultiplier must be solved to realize a compact and low-cost urea sensor system that can be installed in a dialysis machine. An attempt was made to increase the CL emission so as to measure CL using a low-cost photodiode (PD) and to make a reactor that is highly durable. In this paper, we will present the design of the improved urea sensor and its features.

\section{Experimental Methods}

\subsection{Experimental apparatus}

First, a flow injection system composed of a microreactor and two pumps was used in the preliminary experiment. Figure 1(a) shows the microreactor (A-type) for measuring the total CL emission from 7 cells of $3 \mathrm{~mm}$ diameter fabricated in a plate of acrylic resin. A photosensor module (PM; H10722-110 Hamamatsu, Japan) composed of a photomultiplier tube (PMT) and an amplifier unit (whose gain was set to $2 \times 10^{6}$ ) was placed on the reactor. The output voltage of the PM was recorded on a SD card using the H8SX/1655 microcomputer (Renesas Electronics, Japan). Sample and reagent solutions were mixed and passed through the reactor using two unimorph pumps (UPS-112G Nitto Kohki, Japan). An orifice was used to control the flow rate of the reagent. The flow rates of the sample solutions $(\mathrm{S})$ and reagent $(\mathrm{R})$ in the microreactor were 36.9 and $10.6 \mathrm{~mL} / \mathrm{min}$, respectively.

To investigate the $\mathrm{CL}$ from each cell of the A-type reactor, we fabricated a measuring system with a microreactor composed of seven microcells ( $3 \mathrm{~mm}$ i.d., $3 \mathrm{~mm}$ depth) connected 


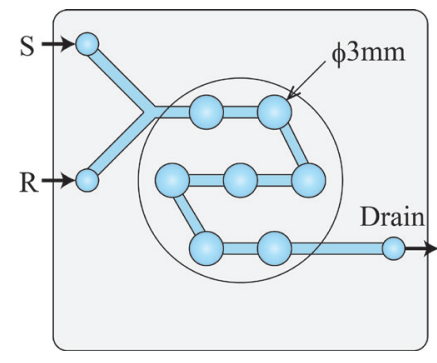

(a)

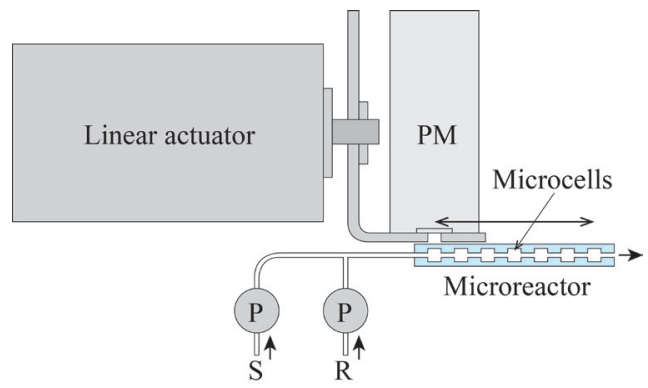

(b)

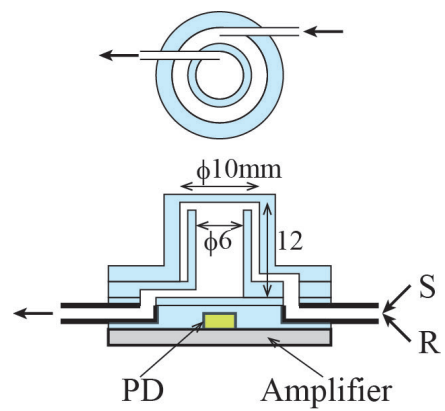

(c)

Fig. 1. (Color online) Schematic illustrations of three types of reactors for measuring CL produced by the reaction of urea with hypobromite. (a) A-type microreactor for measuring the total CL emission from seven microcells (3 $\mathrm{mm}$ i.d.,3 mm depth). (b) System for measuring CL emission from each microcell of the same dimensions as the A-type reactor. (c) B-type reactor that produces a vortex of reacting solution.

in a straight line with tubes of $1 \mathrm{~mm}$ i.d. in a plate of acrylic resin, as shown in Fig. 1(b). The PM (H10722-110 Hamamatsu, Japan) was placed on a masking plate with a window of $3 \mathrm{~mm}$ diameter, and the plate was moved along the microcells using a linear actuator (SCN5 Dyadic, Japan). The MP-1000 peristaltic pump (EYELA, Japan) was used to deliver flow streams of the sample and reagent. The flow rates of the solutions $S$ and $R$ were controlled at a ratio of 4:1.

Figure 1(c) shows a new B-type reactor designed to emit a large amount of CL. We created a prototype of a compact low-cost urea sensor system using the B-type reactor for monitoring urea concentrations in the spent dialysate during dialysis treatment, as shown in Fig. 2. The spent dialysate is sampled from the drain of the dialysis machine TR-2000M (Toray Medical, Japan) used and flows into a buffer tank (100 $\mathrm{mL}$ in volume) in the urea sensor to remove gas bubbles in the spent dialysate. Diaphragm pumps PS (Denso-sangyo, Japan) and PR (Misumivona, Japan) are used to deliver the sample in the tank and reagent solutions in a flexible bag, respectively. This solution is introduced into a concentric cylindrical reactor $(12 \mathrm{~mm}$ i.d., 12 $\mathrm{mm}$ deep) made of acrylic resin configured such that the solution is mixed by a vortex flow. The pumps PS and PR are controlled at flow rates of 300 and $75 \mathrm{~mL} / \mathrm{min}$, respectively, using flow sensors. The CL from the reactor is detected using a Si PD, and the output current is measured using a digital voltmeter through a current amplified gain of $48 \mathrm{mV} / \mathrm{pA}$. The external dimensions of the urea sensor system are $120 \times 170 \times 150 \mathrm{~mm}^{3}$, making the system small enough to be built into a dialysis machine.

\subsection{Reagents}

All chemicals used were of reagent grade (Kanto Chemical, Japan) unless stated otherwise. $5.0 \%$ (as available chlorine) sodium hypochlorite ( $\mathrm{NaClO}$, a special-grade reagent, Kanto Chemical, Japan) solution was used and its factor F was measured using a DPD free-chlorine reagent. The aqueous solution of reagent $\mathrm{A}$ was prepared by dissolving 2.1 mol of sodium 


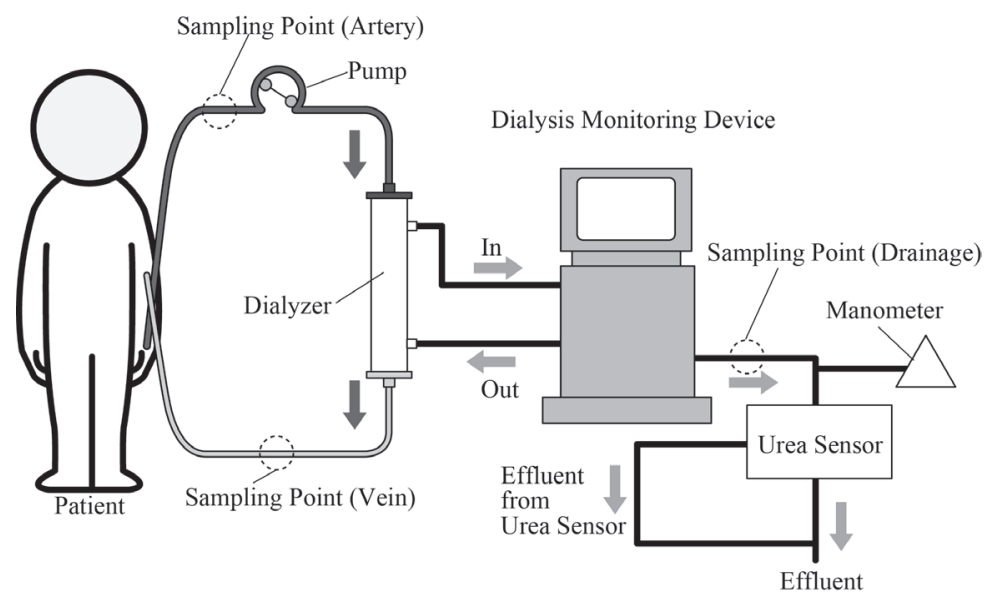

Fig. 2. Schematic illustrations of urea sensor system using the B-type reactor for monitoring urea concentrations in spent dialysate during dialysis treatment.

bromide $(\mathrm{NaBr})$ and $0.8 \mathrm{~mol}$ of sodium hydroxide $(\mathrm{NaOH})$ in water with a total volume of $0.5 \mathrm{~L}$. The solution of reagent $\mathrm{B}$ was prepared by mixing $0.387 / \mathrm{F} \mathrm{L}$ of $5 \% \mathrm{NaClO}$ solution with sterile purified water with a total volume of $0.5 \mathrm{~L}$. Reagent B was stored in a refrigerator and used within $7 \mathrm{~d}$ of preparation. The solution of oxidizing reagent $\mathrm{R}$ was prepared by mixing reagents $\mathrm{A}$ and $\mathrm{B}$ just before use in a urea sensor.

\section{Results and Discussion}

\subsection{CL measurements using a microreactor}

$\mathrm{Hu}$ et al. ${ }^{(9,10)}$ reported that CL reaches a maximum intensity at $20 \mathrm{~ms}$ and is extinguished within $400 \mathrm{~ms}$ after the urea CL in the measurement with a stopped-flow system using a scroll Pyrex glass tube ( $2 \mathrm{~mm}$ i.d.). They proposed the following scheme of the chemiluminescence reaction:

$$
\begin{gathered}
\left(\mathrm{NH}_{2}\right)_{2} \mathrm{CO}+2 \mathrm{OBr}^{-} \rightarrow \mathrm{N}_{2}{ }^{*}+2 \mathrm{Br}^{-} \\
\mathrm{N}_{2}{ }^{*} \rightarrow \mathrm{N}_{2}+h v .
\end{gathered}
$$

In fact, in our test experiments using a similar scroll-type reactor, we found that stable and reproducible CL could not be observed because of the generation of gas bubbles in the reactor during continuous measurements of urea in the spent dialysate sampled during the dialysis treatment of a patient. Thus, we conducted CL measurements using the A-type microreactor of less reagent consumption shown in Fig. 1(a) in the preliminary experiment, because we found that tandem microcells are suitable for producing radical turbulence for quickly mixing solutions that emit CL at a very high reaction rate and generate gas bubbles. First, we investigated the appropriate composition of the oxidizing reagent that reacts with urea to produce CL emissions. 
Figure 3(a) shows the output voltage $V_{S}$ of the PM detecting CL emissions as a function of the concentration of $\mathrm{NaOH}$ in reagent $\mathrm{R}$. $\mathrm{NaClO}$ and $\mathrm{NaBr}$ were kept at constant component concentrations of 0.3 and $1.2 \mathrm{M}$, respectively, and reacted with dialysate D containing $5 \mathrm{mM}$ urea. $V_{S}$ increases with the $\mathrm{NaOH}$ concentration $C_{H}$ in reagent $\mathrm{R}$ and saturates in the region above $0.8 \mathrm{M}$, so that $C_{H}=0.8 \mathrm{M}$ was chosen as the appropriate concentration of $\mathrm{NaOH}$.

Figure 3(b) shows $V_{S}$ as a function of the concentration of $\mathrm{NaBr}$ in reagent $\mathrm{R}$, in which $\mathrm{NaClO}$ and $\mathrm{NaOH}$ were kept at constant component concentrations of 0.3 and $0.8 \mathrm{M}$, respectively, and reacted with the dialysate containing $5 \mathrm{mM}$ urea. $V_{S}$ increases with the $\mathrm{NaBr}$ concentration $C_{B}$ in reagent $\mathrm{R}$ and saturates in the region above $2.1 \mathrm{M}$; thus, $C_{B}=0.8 \mathrm{M}$ was chosen as an appropriate concentration of $\mathrm{NaBr}$.

Figure 3(c) shows $V_{S}$ as a function of the concentration of $\mathrm{NaClO}$ in reagent $\mathrm{R}$, in which $\mathrm{NaBr}$ and $\mathrm{NaOH}$ were kept at constant component concentrations of 2.1 and $0.8 \mathrm{M}$, respectively, and reacted with the dialysate containing $5 \mathrm{mM}$ urea. $V_{S}$ increases with the $\mathrm{NaClO}$ concentration

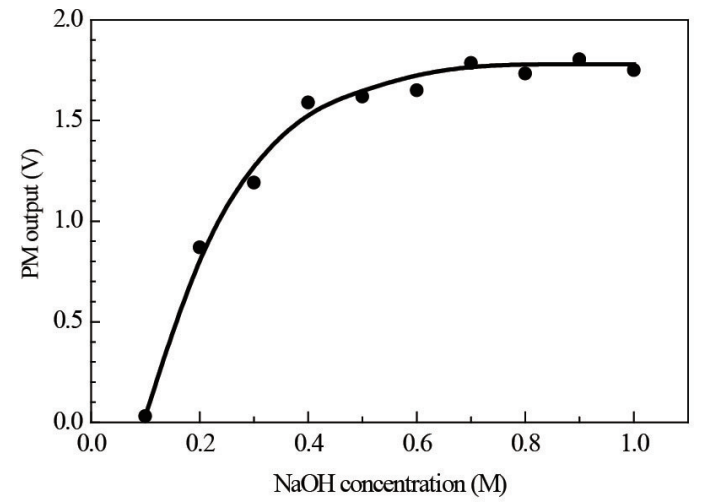

(a)

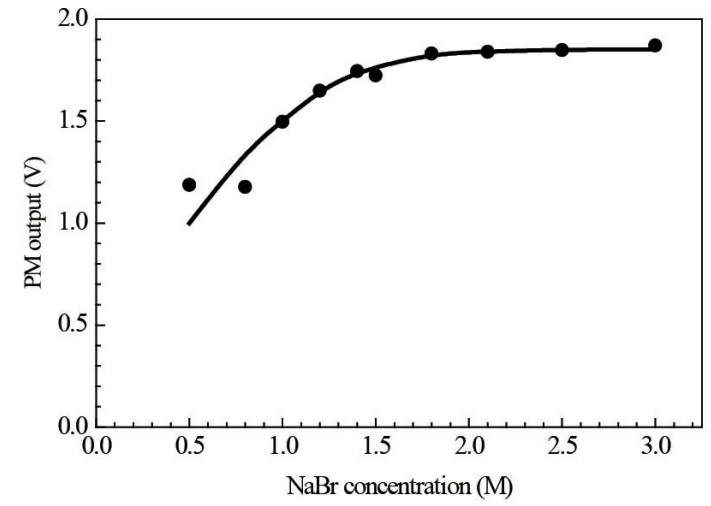

(b)

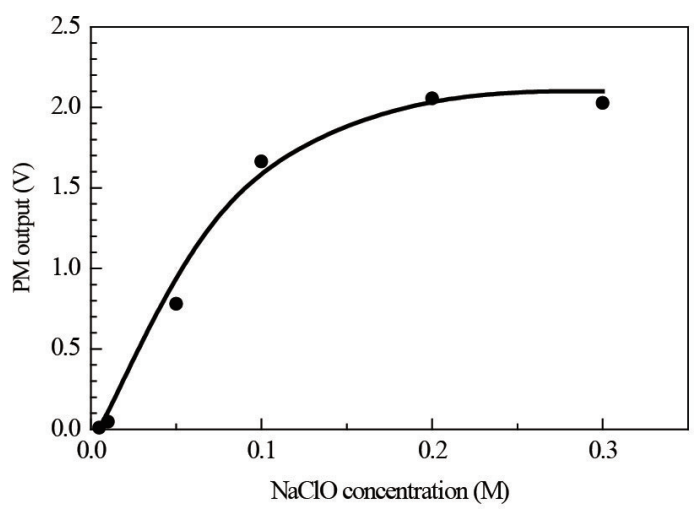

(c)

Fig. 3. Output voltage of the urea sensor as a function of the concentration of the components of reagent R: oxidizing reagent that reacts with urea to produce CL emission. (a) $\mathrm{NaOH}$ concentration dependence, (b) $\mathrm{NaBr}$ concentration dependence, and (c) $\mathrm{NaClO}$ concentration dependence. 
$C_{C}$ in reagent $\mathrm{R}$ and almost saturates at $0.3 \mathrm{M}$; thus, $C_{C}=0.3 \mathrm{M}$ was chosen as the appropriate concentration of $\mathrm{NaClO}$. Therefore, reagent $\mathrm{R}$ consisting of a composition of $0.8 \mathrm{~mol}$ of $\mathrm{NaOH}, 0.3$ $\mathrm{mol}$ of $\mathrm{NaClO}$, and $2.1 \mathrm{~mol}$ of $\mathrm{NaBr}$ per liter was used in the latter experiments.

Figure 4 shows the reproducibility of CL measured over $4 \mathrm{~h}$ for the dialysate containing 5 $\mathrm{mM}$ urea. In this measurement, the sample solution was passed through the reactor for $60 \mathrm{~s}$, then the reagent solution was passed through for $30 \mathrm{~s}$ every 5 min to prevent the contamination of the reactor. The average PM output $V_{S}$ over $15 \mathrm{~s}$ was recorded. The relative standard deviation of $V_{S}$ measured over $4 \mathrm{~h}$ was $1.4 \%$, which confirms the stability of the urea sensor using this microreactor.

\subsection{Improved reactor emitting intense $\mathbf{C L}$}

A low-cost and compact urea sensor system is required to realize urea monitoring during a dialysis session using a dialysis machine incorporating a urea sensor. For this purpose, we investigated an improved reactor emitting an intense CL detectable using a photodiode (PD).

Figure 5 shows the CL emission measured from each microcell separately using the microreactor shown in Fig. 1(b). The flow rates of S and R were kept at a ratio of 3.5:1, and the CL intensities measured under the total flow rates of 20 (filled circles), 30 (filled triangles), and 40 (filled squares) $\mathrm{mL} / \mathrm{min}$ were plotted on the semilog graph. As the retention time $t_{r}$ of the solution in each cell is set to $t_{r}=V_{c} / Q$, where $V_{c}$ is the cell volume $\left(21.2 \mathrm{~mm}^{3}\right)$ and $Q$ is the flow rate, the horizontal axis, i.e., the number $n$ of each cell, can be replaced by time $t=t_{r} n$ in Fig. 5. Therefore, in Fig. 5, the straight lines A, B, and C are calculated with the equation $I=I_{0} \exp (-k t)$, where $I$ is the CL intensity and $k$ is the rate constant of the reaction generating CL. Although the plots are deviated, the value of $k=14 \mathrm{~s}^{-1}$ and the time constant $\tau$ of the CL decay curve, $\tau=1 / k=0.072 \mathrm{~s}$, were obtained by fitting the calculated values with plots in Fig. 5 .

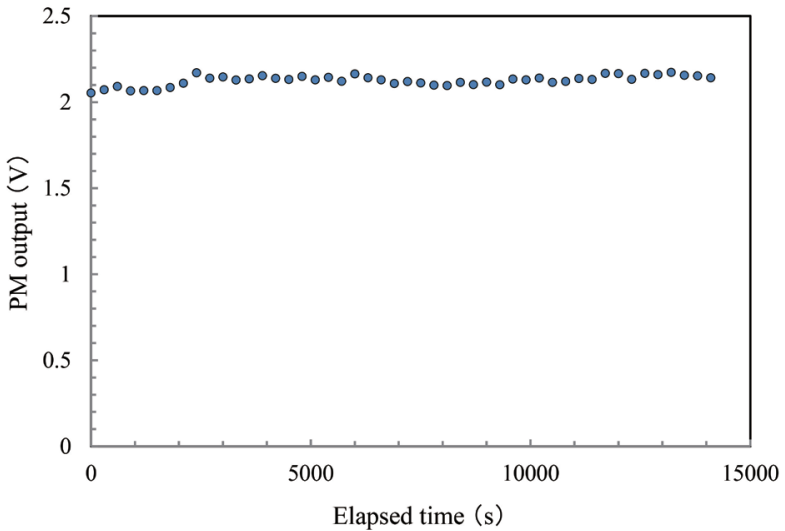

Fig. 4. (Color online) Reproducibility of output voltage of the urea sensor for dialysate containing 5 $\mathrm{mM}$ urea.

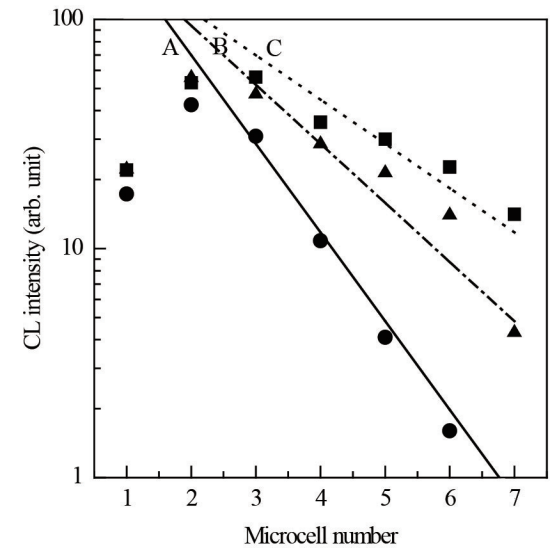

Fig. 5. Intensity of CL emission from each microcell measured separately using the reactor shown in Fig. 1(b). The CL intensities were measured under the total flow rates of 20 (filled circles), 30 (filled triangles), and 40 (filled squares) $\mathrm{mL} / \mathrm{min}$. 
This $k$ value is not that different from the value of $k=10.9 \mathrm{~s}^{-1}$ reported by $\mathrm{Hu}$ et al. from their experiments on the CL generated by the reaction of urea with hypobromite, even though their concentrations were different from those in our experiments. ${ }^{(10)}$ The total CL emission can be simply estimated by integrating the value of CL emission decay with time over the retention time of the reaction solution in a reactor under the complete mixing condition of the sample and reagent solutions.

On the basis of the results described above, we designed the B-type reactor [Fig. 1(c)] to produce a vortex so as to realize a long retention time of the reacting solution. Figure 6 shows the output voltage $V_{S}$ of a PM attached to the c-type reactor measured as a function of total flow rate $Q$ for CL using $0.083 \mathrm{mM}$ urea solution. $V_{S}$ increases with $Q$ and reaches $2.2 \mathrm{~V}$ at $Q=250$ $\mathrm{mL} / \mathrm{min}$, which corresponds to approximately 60 times that measured using an A-type reactor for the urea solution of the same concentration. Thus, we were able to produce a prototype device with a compact urea sensor using a PD with a high-gain current amplifier of $48 \mathrm{mV} / \mathrm{pA}$ as a CL detector.

\subsection{Characteristics of compact urea sensor}

Figure 7(a) shows the concentration dependence of the sensor output $V_{s}$ measured as a function of the urea concentration $C_{u}$ in an aqueous solution. $V_{s}$ increases linearly with $C_{u}$. The urea concentration in blood (BUN) is usually expressed in units of urea nitrogen (UN) concentration $(\mathrm{mg} / \mathrm{dL})$ in the medical field and defined as

$$
\mathrm{UN} \text { concentration }[\mathrm{mg} / \mathrm{dL}]=C_{u}[\mathrm{~mol} / \mathrm{L}] \times M_{u}[\mathrm{~g} / \mathrm{mol}] \times \frac{M_{N}[\mathrm{~g} / \mathrm{mol}]}{M_{u}[\mathrm{~g} / \mathrm{mol}]} \times 0.1 \text {, }
$$

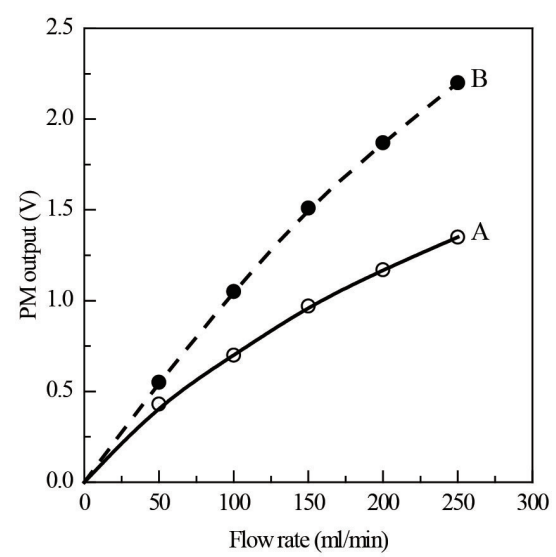

Fig. 6. Output voltage of the compact urea sensor measured as a function of total flow rate in the B-type reactor without (open circles) and with (filled circles) reflector for $0.83 \mathrm{mM}$ urea solution. 


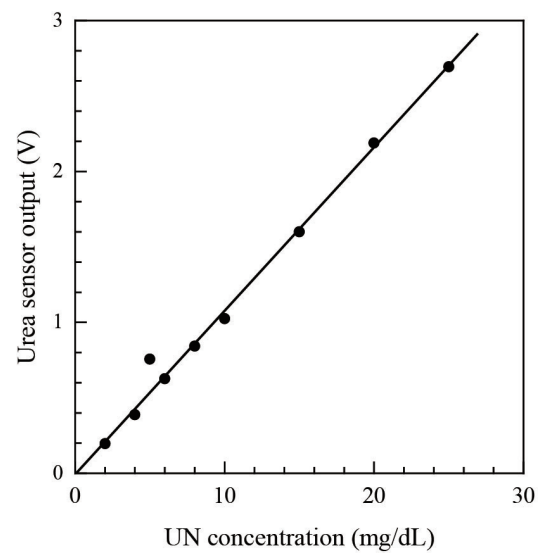

(a)

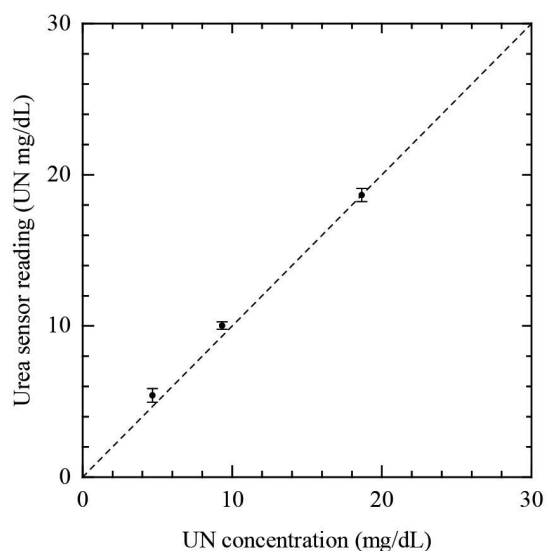

(b)

Fig. 7. Output of the compact urea sensor with B-type reactor. (a) Output voltage VOUT measured as a function of urea concentration in water and (b) reading of the calibrated urea sensor as a function of urea concentration in dialysate. The error bar shows the standard deviation for 20 measurements.

where $C_{u}, M_{u}$, and $M_{N}$ are the concentration of urea in units of $\mathrm{mg} / \mathrm{dL}$, and the molecular weights of urea and nitrogen, respectively. The UN concentration range in the spent dialysate was $3.5-22 \mathrm{mg} / \mathrm{dL}$ for two patients measured during in vivo dialysis treatments under various conditions, ${ }^{(12)}$ and the detection range of the urea sensor is $2-25 \mathrm{mg} / \mathrm{dL}$. Thus, the compact urea sensor system has sufficient sensitivity for the measurement of urea during dialysis treatment.

Figure 7(b) shows the reading of the calibrated urea sensor measured as a function of urea concentration in the dialysate. The error bar shows the standard deviation $\sigma$ for 20 measurements, and the maximum value of $\sigma$ is $0.45 \mathrm{mg} / \mathrm{dL}$.

Figure 8 shows the relative changes in the output of the urea sensor measured as a function of the temperature of the sample solution. The relative temperature coefficient of the sensor output was estimated to be $1.8 \% /{ }^{\circ} \mathrm{C}$ from the slope of the curve in Fig. 8 . However, no temperature compensation is required, because the temperature of the dialysate is controlled at about the same temperature as the blood temperature by the dialysis machine, and the temperature in the dialysis treatment room is usually kept constant.

To investigate the difference in sensor output for urea in the water and dialysate, the effects of acid and electrolytes on $V_{S}$ and $\mathrm{UN}$ concentration measured using two urea sensors, A and B, are shown in Tables 1(a) and 1(b), respectively.

In these measurements, the composition of the electrolyte was as follows: $140 \mathrm{mM} \mathrm{Na}$, $2 \mathrm{mM} \mathrm{K}^{+}, 3 \mathrm{mM} \mathrm{Ca}^{++}, 1 \mathrm{mM} \mathrm{Mg}{ }^{++}, 111 \mathrm{mM} \mathrm{Cl}^{-}$, and $8.3 \mathrm{mM}$ glucose. The concentrations of urea, $\mathrm{HCO}_{3}$, acetic acid, and citric acid in sample solutions were 28.5, 35, 9, and $2 \mathrm{mM}$, respectively. As the shifts of the sensor output and sensor reading are small, errors can be eliminated by day-to-day calibration.

\subsection{In vitro measurements of urea during dialysis session}

Finally, in vitro measurements of urea concentration in the spent dialysate during a dialysis session were conducted using a precalibrated compact urea sensor system. An aqueous solution 


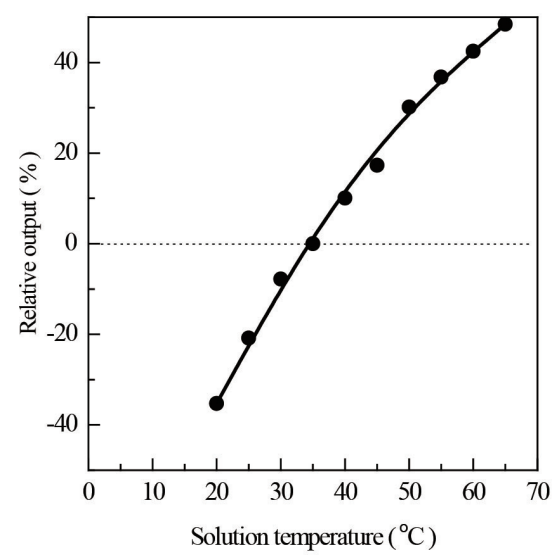

Fig. 8. Relative output of the urea sensor measured as a function of the temperature of $0.83 \mathrm{mM}$ urea solution .

Table 1

Effects of acids and electrolytes on urea sensors.

(a) Effect on output voltage of sensor A.

\begin{tabular}{lccc}
\hline $\begin{array}{l}\text { Solute of sample } \\
\text { solution }\end{array}$ & Urea & Urea $+\mathrm{HCO}_{3}$ & $\begin{array}{l}\text { Urea }+\mathrm{HCO}_{3} \\
+ \text { acetic acid }\end{array}$ \\
\hline Sensor output (V) & 2.99 & 2.97 & 2.79 \\
\hline
\end{tabular}

(b) Effect on the sensor reading [UN concentration $(\mathrm{mg} / \mathrm{dL})]$ for calibrated sensor B.

\begin{tabular}{lcccc}
\hline $\begin{array}{l}\text { Solute of sample } \\
\text { solution }\end{array}$ & Urea & $\begin{array}{c}\text { Urea }+ \\
\text { electrolytes }\end{array}$ & $\begin{array}{c}\text { Urea }+ \text { electrolytes } \\
+\mathrm{HCO}_{3}+\text { acetic acid }\end{array}$ & $\begin{array}{c}\text { Urea + electrolytes } \\
+\mathrm{HCO}_{3}+\text { citric acid }\end{array}$ \\
\hline $\begin{array}{l}\text { Sensor reading } \mathrm{UN} \\
\text { concentration }(\mathrm{mg} / \mathrm{dL})\end{array}$ & 23.1 & 24.0 & 23.9 & 24.0 \\
\hline
\end{tabular}

of $80 \mathrm{mg} / \mathrm{dL}$ UN was prepared as a sample solution for in vitro measurements. The dialysis session for $30 \mathrm{~L}$ of sample solution in a container was conducted for $4 \mathrm{~h}$ using a TR-3000M dialysis machine (Toray, Japan) and an FX-220 dialyzer (Fresenius Medical Care, Japan). Figure 9 shows the reading of the urea sensor $C_{S}$ measured at $10 \mathrm{~min}$ intervals for the spent dialysate (filled circles), and $C_{S}$ is plotted as a function of the elapsed dialysis time $t$ on a semilog graph. In Fig. 9, the plots $C_{D}, C_{A}$, and $C_{V}$ show the $\mathrm{UN}$ concentrations measured at the Okayama Medical Laboratory, Inc. (OML, Japan) by the Urease India Phenol method for the solutions sampled at 30 min intervals from the spent dialysate side (open circles), blood arterial side (open triangles), and blood venous side (open downward triangles), respectively.

The values of $C_{S}$ and $C_{D}$ almost match, but their maximum relative deviation is approximately $15 \%$. On the other hand, the $\log C_{S}, \log C_{D}, \log C_{A}$, and $\log C_{V}$ plotted as a function of $t$ fit into straight lines, and the time constants $\tau$ of decay are 112, 108, 107, and $110 \mathrm{~s}$, respectively, and their relative deviation of $\tau$ is less than $4 \%$. As the urea concentrations in the blood and spent dialysate decrease exponentially with dialysis time under the condition that the 1-pool model is applicable, we can estimate the dialysis efficiency from the decay-time constant $\tau$. ${ }^{(12)}$ These results show that the compact urea sensor system has sufficient reliability to evaluate dialysis efficiency during dialysis treatment. 


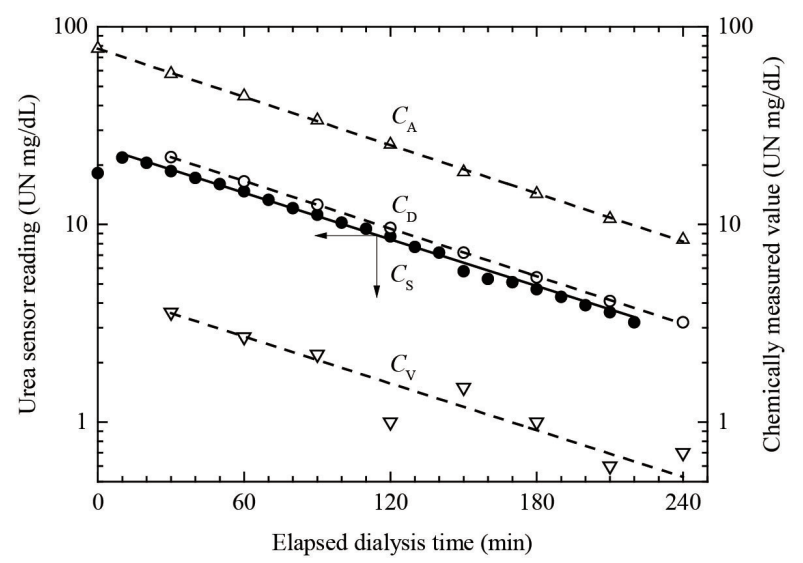

Fig. 9. In vitro measurements of urea during a dialysis session for a sample solution of $80 \mathrm{mg} / \mathrm{dL} \mathrm{UN} 30 \mathrm{~L}$ in volume: reading of the urea sensor (filled circles), UN concentrations measured at the Okayama Medical Laboratory, Inc. by the Urease India Phenol method for the solutions sampled from the spent dialysate side (open circles), blood arterial side (open triangles), and blood venous side (open downward triangles) of the dialysis circuit.

\section{Conclusions}

We developed a prototype of a compact urea sensor system that can be installed in a dialysis machine for monitoring urea concentration during dialysis. The urea sensor was based on the CL due to the reaction of urea with hypobromite. The CL from the new vortex-flow-type reactor was detected using a low-cost compact Si PD (Hamamatsu, Japan) with a high-gain current amplifier of $48 \mathrm{mV} / \mathrm{pA}$, so we could measure urea nitrogen of concentrations between 2 and $30 \mathrm{mg} / \mathrm{dL}$. The urea sensor with such a measuring range had sufficient sensitivity for measuring the change in urea concentration in the spent dialysate $(17-5 \mathrm{mg} / \mathrm{dL})$ during in vivo dialysis treatment. The sensor showed a good repeatability accuracy of $1.9 \%$, and the readings of sensor $C_{S}$ showed good correlation with chemically measured UN concentrations at the Okayama Medical Laboratory, Inc. The external dimensions of the urea sensor system including the power supply, computer, and display unit were $120 \times 170 \times 150 \mathrm{~mm}^{3}$, and the sensing unit was small enough to enable multiple units to be built into a dialysis machine. This sensor system will be used to realize automatic dialysis machines in the near future.

\section{References}

1 F. A. Gotch and J. A. Sargent: Kidney Int. 28 (1985) 526.

2 W. F. Owen, Jr., N. L. Lew, Y. Liu, E. G. Lowrie, and J. M. Lazarus: N. Engl. J. Med. 329 (1993) 1001.

3 R. Koncki, A. Radomska, and S. Glab: Anal. Chim. Acta 418 (2000)213.

4 R. Koncki, A. Radomska, and S. Glab: Talanta 52 (2000) 13.

5 W. Sant, M. L. Pourciel, J. Launay, T. D. Conto, A. Martinez, and P. Temple-Boyer: Sens. Actuators, B 95 (2003) 309.

6 O. A. Boubriak, A. P. Soldakin, N. F. Starodub, A. K. Sandrovsky, and A. K. El'skaya: Sens. Actuators, B 26 (1995) 429.

7 V. R. Kondepati, U. Damm, and H. M. Heise: Appl. Spectrosc. 60 (2006) 920.

8 Z. Filutowicz, K. Lukaszewski, and K. Pieszynski: J. Med. Inf. Technol. 8 (2004) 1642. 
9 X. Hu, N. Takenaka, M. Kitano, H. Bandow, and Y. Maeda: Analyst 119 (1994) 1829.

10 X. Hu, N. Takenaka, M. Kitano, H. Bandow, and Y. Maeda: Bull. Chem. Soc. Jpn. 69 (1996) 1179.

11 M. Ozaki, T. Okabayashi, T. Ishimaru, K. Hayashi, J. Hori, I. Yamamoto, and M. Nakagawa: IEEJ Trans. SM 130 (2010) 11.

12 M. Ozaki, J. Hori, and T. Okabayashi: Ther. Apher. Dial. 18 (2014) 193.

\section{About the Authors}

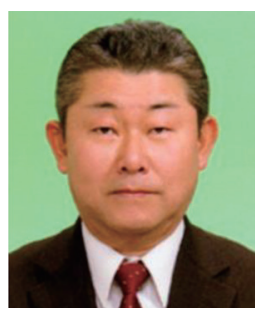

Kunihito Hayashi received his B.S. degree from Kawasaki College of Allied Health Professions, Japan, in 1991 and his M.S. degree from the Okayama University of Science, Japan, in 2017. Since 2005, he has been a clinical engineer at Sumida Clinic, Japan. He is interested in developing medical devices related to hemodialysis. (Kunihitom@mx3.kct.ne.jp)

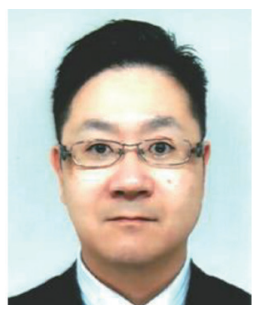

Jun'ya Hori received his M.S. and Ph.D. degrees from Hiroshima University, Japan, in 2000 and 2002, respectively. From 2002 to 2003, he was a lecturer at Hiroshima University, Japan. From 2003 to 2017, he was a lecturer at Okayama University of Science. Since 2017, he has been an associate professor at Okayama University of Science. His research interests are in (a) the safety management of medical electrical equipment, for example, defibrillators and AEDs, and electrosurgical knives and (b) real-time urea sensor for hemodialysis treatment using the chemiluminescence phenomenon. (j-hori@dap.ous.ac.jp)

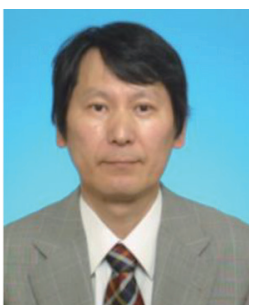

Masahiro Ozaki received his Ph.D. degree from the Okayama University of Science, Japan, in 2013. From 1988 to 2005, he was a chief clinical engineer at Shigei Medical Research Hospital, Japan. Since 2013, he has been a professor at Okayama University of Science. He is interested in developing medical equipment. (ozaki@dap.ous.ac.jp)

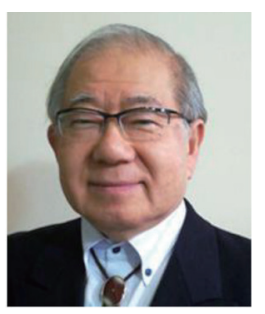

Masuo Nakagawa received his M.S. and Ph.D. degrees from Kwansei Gakuin University, Japan, in 1970 and 1983, respectively. From 1997, he was a professor at Okayama University of Science, Japan. Since 2015, he has been a professor emeritus. He is interested in electronic and optical phenomena at interfaces, and their medical applications using chemical sensors. (masuoxxxxxnaka@gmail.ac.jp) 


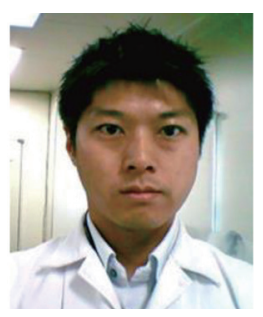

Masfumi Hashiguchi received his B.S. degree from Tokyo University of Science, Japan, in 2005. From 2005 to 2009, he was in charge of the electrical design of the Push Engine Start System for automobiles at Alpha Corporation. Since 2009, he has been with the Industrial Technology Development Center of Ricoh Company Ltd. He is in charge of the development of optical sensing modules for measuring apparatus and electrophotographic image-forming apparatus. His research interests are in applications of analog sensing. (masafumi.hashiguchi@nts.ricoh.co.jp) 